\title{
CONDITIONAL RELEASE OF NEW ORGANISMS IN NEW ZEALAND
}

\author{
C.M.C. VIEGLAIS ${ }^{1}$ and L. HARRISON ${ }^{2}$ \\ ${ }^{1}$ MAF Biosecurity Authority, Plants Biosecurity, P.O. Box 2526, Wellington, \\ New Zealand \\ ${ }^{2}$ Environmental Risk Management Authority, P.O. Box 131, Wellington, \\ New Zealand \\ Corresponding author: VieglaisC@maf.govt.nz
}

\begin{abstract}
New organisms are defined as species not present in New Zealand prior to 29 July 1998, the date the New Organisms component of the Hazardous Substances and New Organisms (HSNO) Act 1996 came into effect. New organisms also include genetically modified (GM) organisms, organisms that have been approved for containment or conditional release, and exotic organisms that have been eradicated. There are three ways a new organism can occur in New Zealand: accidental incursions, deliberate but illegal introductions, and legal introductions. Applications for introductions are evaluated by the Environmental Risk Management Authority (ERMA New Zealand). In October 2003, the HSNO Act was amended to allow for the conditional release of new organisms, including GM organisms. This paper describes the process for the introduction of new organisms into New Zealand, with an emphasis on conditional release to assess the risks, costs and benefits of new organisms to human health, the environment and the economy.

Keywords: Ministry of Agriculture and Forestry, Environmental Risk Management Authority, new organism, genetic modification, conditional release.
\end{abstract}

\section{INTRODUCTION}

New Zealand is economically dependent on introduced organisms for its horticulture, agriculture and forestry industries. The production and processing of exotic plants, animals and trees contributes to over $60 \%$ of New Zealand's export earnings and over $10 \%$ of the national gross domestic product of $\$ 116$ billion ending 30 June 2003 (Statistics NZ 2004). In addition to the enormous economic contributions of introduced biota to New Zealand, some exotics have also caused considerable harm to the environment (e.g. possum, varroa mite and gorse). Despite this, introductions of new organisms are likely to continue to provide an important input to New Zealand's primary productivity. In order to protect the environment, and the health and safety of people and communities, it is essential that the risks, costs and benefits of any new organism be evaluated prior to its full release.

To prevent future negative impacts from new organisms, New Zealand's borders are well controlled and organisms that enter legally do so under considerable scrutiny. Under the Biosecurity Act 1993, the Ministry of Agriculture and Forestry (MAF) provides border surveillance to prevent accidental incursions and deliberate but illegal introductions of unwanted organisms. The Hazardous Substances and New Organisms (HSNO) Act 1996 is the principal law for managing the legal introduction of new organisms. HSNO established the Environmental Risk Management Authority (ERMA New Zealand) which evaluates all applications for the import, development, field testing or release of new organisms. ERMA liaises frequently with MAF, which is the enforcement agency for the New Organisms component of the HSNO Act. 
The objective of this joint MAF-ERMA review paper is to provide guidance for interested parties seeking approvals to introduce new organisms into New Zealand. Plant protection scientists make up a large pool of applicants for new organisms applications (ERMA NZ 2004a). Submissions include novel germplasm to aid in breeding diseaseand pest-resistant plants; genetically modified plants containing selected traits of importance to New Zealand farmers and growers; and biocontrol agents to counteract weeds, pests and diseases.

\section{New Organism Status}

Before applying to ERMA for a new organism approval, applicants should confirm that their organism is in fact "new". In general, new organisms are defined under the HSNO Act on the basis of when they first arrived to New Zealand, how they came into existence and under what conditions they are to be used (Table 1). In addition to applying the definitions found in the HSNO Act to determine if an organism is new, a statutory register of new organisms already approved for importation can be found on ERMA's website and should be consulted (ERMA NZ 2004a). If the organism is a plant, the Plant Biosecurity Index (MAF 2004) may also provide information regarding whether the species is already in New Zealand. However, the final determination rests with ERMA as to whether the organism is new and requires import approval under HSNO, or whether it is not new and therefore is only subject to MAF's import provisions under the Biosecurity Act.

\section{TABLE 1: Definitions in the HSNO Act ${ }^{1}$ (Sec. 2) pertaining to new organisms.}

Organism does not include a human being, but does include a human cell, a micro-organism, or a genetic structure other than a human cell that is capable of replicating itself.

Containment means restricting an organism to a secure location or facility to prevent escape; and includes, in respect of genetically modified organisms, field testing and large scale fermentation.

Release means to allow the organism to move within New Zealand. A full release is free of any restrictions other than those imposed in accordance with the Biosecurity Act 1993 or the Conservation Act 1987, whereas a conditional release includes controls.

Control means any obligation or restriction imposed on any new organism or any person in relation to any new organism for the purposes of controlling the adverse effects of that organism on people or the environment.

Field test means the carrying on of trials on the effects of the organism under conditions similar to those of the environment into which the organism is likely to be released, but from which the organism, or any heritable material arising from it, could be retrieved or destroyed at the end of the trials.

Develop means to genetically modify, regenerate from biological material that cannot be used to reproduce the organism without human intervention, or ferment a new organism in containment such as a laboratory; does not include field testing.

Genetically modified organism means any organism or its progeny in which any genetic material has been modified by in vitro techniques.

New Organism means:

(a) a species not present in New Zealand immediately prior to 29 July 1998, the date the New Organisms component of the HSNO Act came into effect;

(b) an organism prescribed as a risk "species", where any member of a taxonomic classification of that species - subspecies, infrasubspecies, variety, strain or cultivar may have adverse effects on the health and safety of people or the environment;

(c) an organism for which a containment or a conditional release approval has been given;

(d) a qualifying organism (an organism contained in a medicine or veterinary medicine) approved for release with controls;

(e) a genetically modified organism; and

(f) an organism that has been eradicated from New Zealand.

${ }^{1}$ Definitions extracted from Hazardous Substances \& New Organisms Act 1996, No. 30, as amended (HSNO 1996). 


\section{Categories of approvals}

A new organism can be approved for import into containment (development or field test) or for release (conditional or full). There is no statutory requirement for the approval to proceed in any particular order through the stages from development to full release. In fact, because full releases are not considered new organisms, it is conceivable that an organism that meets the definition of "new", yet is deemed safe enough to be approved for import directly to full release, would never technically exist in New Zealand as a new organism. However, it is anticipated that for most new organisms, there will be a period of containment or conditional release (or both) before a full release could be justified. The addition of the conditional release category in amendments to HSNO in Oct 2003 significantly expanded the possibilities for studying new organisms. Whereas field tests are strictly contained, regulated, and monitored, conditional releases are not contained and the degree of regulation and monitoring is flexible. This allows for a much broader range of circumstances under which to assess the impacts of new organisms. With conditional releases, controls are designed to manage risk yet allow comprehensive, high quality information to be gathered on the environmental, cultural, economic, social and other effects of the new organism.

\section{Responsibilities}

The purpose of the HSNO Act is "to protect the environment, and the health and safety of people and communities, by preventing or managing the adverse effects of hazardous substances and new organisms." When preparing an application, the applicant needs to carefully consider their statutory responsibilities in two areas relevant to the purpose of the Act (Sec. 5 \& 6):

5. "...recognise and provide for the following principles:

(a) The safeguarding of the life-supporting capacity of air, water, soil, and ecosystems:

(b) The maintenance and enhancement of the capacity of people and communities to provide for their own economic, social, and cultural wellbeing and for the reasonably foreseeable needs of future generations."

6. "...take into account the following matters:

(a) The sustainability of all native and valued introduced flora and fauna:

(b) The intrinsic value of ecosystems:

(c) Public health:

(d) The relationship of Maori and their culture and traditions with their ancestral lands, water, sites, waahi tapu, valued flora and fauna, and other taonga:

(e) The economic and related benefits [and costs of using] a particular hazardous substance or new organism:

(f) New Zealand's international obligations"(HSNO 1996).

\section{Identification of risks, costs and benefits}

The applicant has primary responsibility for identifying and assessing risks, while ERMA has responsibility for evaluating risk and making decisions based on combined consideration of risks, costs, and benefits (ERMA NZ 2004b). For conditional releases, it is most important for the applicant to analyse at the outset the ability of the organism(s) to establish a self-sustaining population and the ease with which the organism(s) could be recovered or eradicated if an undesirable self-sustaining population established. Important criteria to consider are the natural habitat, life cycle, distribution, history of introduction outside their natural range and natural competitors of the organism. Effects of the new organism on the environment, human health and safety, relationship of Maori and their culture, economy, culture, society, ethics and spiritual issues must also be systematically assessed.

An application for a conditional release requires considerably more detail than that for a development or field test because of the greater possibility for adverse effects 
outside of containment. In the preapplication period, it is strongly recommended that applicants discuss their draft application with ERMA before it is formally submitted (ERMA NZ 2004c). Providing notification for public and Maori consultation during the decision-making process is required and ERMA must be informed of proprietary information beforehand.

\section{DECISION-MAKING CRITERIA}

The HSNO Act (Sec. 36) sets clear minimum standards for approvals. An application for a new organism will be declined "if the new organism is likely to -

(a) Cause any significant displacement of any native species within its natural habitat; or

(b) Cause any significant deterioration of natural habitats; or

(c) Cause any significant adverse effect to New Zealand's inherent genetic diversity; or

(d) Cause disease, be parasitic, or become a vector for human, animal or plant disease, unless the purpose of that importation or release is to import or release an organism to cause disease, be a parasite or a vector for disease" (HSNO 1996).

ERMA will consider controls that minimise the environmental, health, economic, cultural, social or other risks associated with the conditional release of a new organism (Table 2).

\section{TABLE 2: Suggested controls for conditional release approvals (Sec 38D) (HSNO 1996).}

(a) controlling the extent and purposes for which organisms could be used

(b) requiring any monitoring, auditing, reporting, and record-keeping

(c) imposing any obligation to comply with relevant codes of practice or standards (for example, to meet particular co-existence requirements)

(d) requiring contingency plans to be developed to manage potential incidents

(e) limiting the dissemination or persistence of the organism or its genetic material in the environment

(f) requiring the disposal of any organisms or genetic material

(g) limiting the proximity of the organism to other organisms, including those that could be at risk from the conditionally released organism

(h) setting requirements that must be met for any material derived from the organism

(i) imposing obligations on the user of an approval, including levels of training or knowledge, limits on the numbers of users who may hold an approval, and the persons that they could deal with in respect of the organism

(j) specifying the duration of the approval or of a control before requiring review

ERMA has developed a series of guides for applicants as well as for the public to provide insight into the approval process for new organism importation, containment and release (ERMA NZ 2004c, 2003a, 2003b, 2003c, 2003d). Once an application is approved with controls, MAF will initiate an inspection regime to monitor compliance.

\section{CONCLUSION}

Through the conditional release process, a system is in place to study the effects of new organisms on the environment, human health and other matters of concern. In keeping with the recommendations of the Royal Commission on Genetic Modification (2001), New Zealand has proceeded carefully, minimising and managing risks, while preserving opportunities. MAF and ERMA welcome the opportunity to facilitate quality research on new organisms while safeguarding the environment, protecting health and encouraging the advancement of knowledge. 


\section{REFERENCES}

ERMA NZ 2003a: Information Sheet No. 19, November 2003, Conditional Release, ER-IS-19-1 11/03 http://www.ermanz.govt.nz/resources/publications/pdfs/ER-IS19-1.pdf (28/05/04).

ERMA NZ 2003b: Quick Guide Enforcement \& Compliance for New Organisms, EROG-14-2 11/03 http://www.ermanz.govt.nz/resources/publications/pdfs/ER-QG-143.pdf (28/05/04).

ERMA NZ 2003c: Information Sheet No. 4, November 2003, New Organisms under the HSNO Act, ER-IS-4-02 11/03 www.ermanz.govt.nz/ (28/05/04).

ERMA NZ 2003d: Quick Guide Applying for New Organism Approval http:// www.ermanz.govt.nz/resources/publications/pdfs/ER-QG-18-3.pdf (28/05/04).

ERMA NZ 2004a: Organism Register. http://www.ermanz.govt.nz/search/organism1.cfm $(28 / 05 / 04)$.

ERMA NZ 2004b: Decision Making: A Technical Guide to Identifying, Assessing and Evaluating Risks, Costs and Benefits, ISBN 0-478-21523-1 http:// www.ermanz.govt.nz/resources/publications/pdfs/er-tg-05-01 $0304 \mathrm{dm}$ tech gde.pdf $(28 / 05 / 04)$.

ERMA NZ 2004c: ERMA New Zealand User Guide to making an application to import for release or release from containment with controls any new organism under Section 38A of the Hazardous Substances and New Organisms Act 1996. http:// www.ermanz.govt.nz/resources/publications/pdfs/ER-UG-NOCR-1.pdf (28/05/04).

HSNO 1996: Hazardous Substances and New Organisms Act 1996, no. 30, as amended. http://www.legislation.govt.nz/browse_vw.asp?content-set=pal_statutes (28/05/04).

MAF 2004. Plants Biosecurity Index http://www1.maf.govt.nz/cgi-bin/bioindex/ bioindex.pl (28/05/04).

Royal Commission on Genetic Modification 2001: The Report of the Royal Commission on Genetic Modification ISBN 0-477-01943-9 http://www.gmcommission.govt.nz/ RCGM/rcgm_report.html (28/05/04).

Statistics NZ 2004: 2003 Agriculture Production Survey. http://www.stats.govt.nz/ domino/external/web/Prod_Serv.nsf/htmldocs/Agriculture,+Forestry+ and+Fishing\#Stats (28/05/04). 